\title{
An Adolescent with innumerable myomas
}

\author{
Baral J., Gurung G. , Mishra S. , Rai N. , Paudyal P. , Shrestha B. , Rana A. \\ Dept of ObGyn, TUTH, Ktm, Nepal \\ Correspondence Address: Dr. Josie Baral, Dept of ObGyn, TUTH, Ktm, Nepal
}

\begin{abstract}
Myoma is rare in younger age group, so tends to be mistaken for adnexal tumour. A 19 year old adolescent who was taken for laparotomy for ovarian germ cell tumour instead was found to have uterus studded with innumerable myomas, which were surgically removed through tedious yet successful myomectomy of 40 myomas, thus deserving description.
\end{abstract}

Key words: Adolescents, myoma, myomectomy

\section{Introduction}

Leiomyomas of the uterus in age under 20 is rare and occurs less frequently than adnexal tumour. ${ }^{1}$ For this reason, whenever a young girl presents with abdominal mass, the first provisional diagnosis made is the germ cell tumour of ovary as in our case. ${ }^{2}$ Even the CT Scan could not predict the questionable tumour mass to be myoma at the outset. This case is fewer among the few, because of having exceptionally large numbers of myomas in young adolescent besides being primarily confused with ovarian tumour, with successful myomectomy operation removing 40 myomas quickly through a tedious process without requiring blood transfusion. ${ }^{3}$

\section{Case}

A 19 year old unmarried girl presented to us in the Gynaecology OPD with complaints of premenstrual dull aching pain in lower abdomen for the past four years along with the mass in the lower abdomen for one month duration. She had a regular menstrual cycle and normal flow she started having dull aching pain in lower abdomen especially 10-12 days before menstruation, from the age of 15 (after 1 year of menarche). Although the pain was not hampering her daily activities and was relieved on taking anti-spasmodic occasionally, yet she went to private clinic for ultra sound, which showed normal abdomen and pelvis. After 3 years, she attended our hospital, with the same complaint. An USG advised, once again showed normal results. This time, one month before she attended our hospital again, her mother had felt a lump in the daughter's lower abdomen while sleeping together. USG done, thereafter showed a mass in the lower abdomen diagnosed as ? uterine / ovarian mass in origin (that brought her back to this hospital.

General and systemic examination was normal. There was huge mass, occupying left lower quadrant of the abdomen, about $20 \mathrm{~cm} \times 15 \mathrm{~cm}$ in size, irregularly enlarged with ill defined margin, firm in consistency freely mobile laterally without tenderness. As there is no sexual exposure bimanual internal pelvic examination $(\mathrm{P} / \mathrm{V})$ was not. $\mathrm{CT}$ scan confirmed poorly defined lobulated heterogeneously enhancing soft tissue density mass in abdomenopelvic cavity with large solid component and few areas of attenuation (Fig. 1).

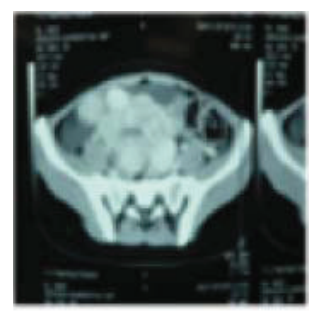

Fig. 1 CT Scan showing multiple mass filling abdomen and pelvis.

Differential diagnosis of ovarian malignancy or neurogenic tumour arising from pelvis was considered. Tumour markers like Ca125 - 26.3, CEA 1.45, Alpha FP - 1.2 and serum beta $\mathrm{hCG}-1.3$ done were within normal limit)

Exploratory laparotomy was done on 16th Jan 2012 under spinal anaesthesia by giving sub umbilical low mid line incision. Intra operative finding showed normal bilateral 
ovaries and tubes but unexpectedly enlarged uterus measuring $26 \times 28 \mathrm{~cm}$ studded with multiple intramural and sub serous (Fig.2).

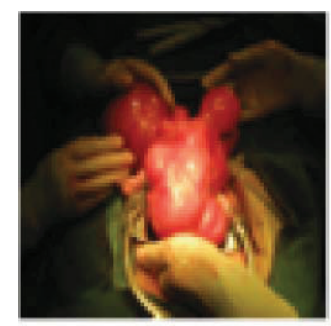

Fig 2. Intraoperative finding of multiple myoma

Before heading for myomectomy, her parents were explained that she might need hysterectomy during the procedure in case of difficulty. Myomectomy was successfully accomplished with the complete removal of 40 differently sized myomas. (Fig. 3) largest being 8x6 cm, cut section being consistent with whorled appearance. Post operatively uterus appeared 12 weeks size.(Fig 4).

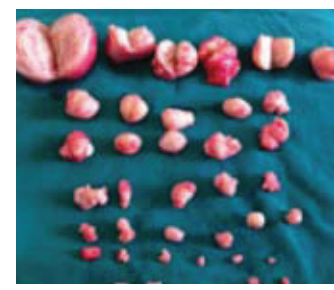

Fig 3. Intraoperative finding of multiple myoma

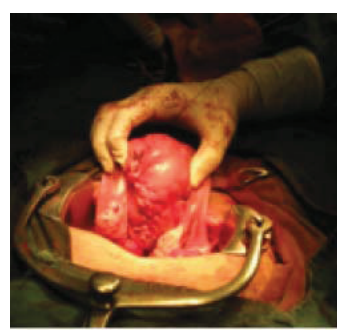

Fig 4. Myoma 40 in number are seen

Time taken for surgery was one and half hours, requiring no blood transfusion, blood loss being $100 \mathrm{ml}$, minimized by vaginal application of misoprostol $800 \mathrm{mcg}$, inserted just before myomectom. Post operative $\mathrm{Hb}$ was $11 \mathrm{gm} \%$ and the post operative period was uneventful, young girl being discharged on the 5th post operative day. She was counselled about pregnancy and caesarean delivery. Histopathology report confirmed myoma.

\section{Discussion}

Uterine leiomyoma is rarely seen in the pediatric and adolescent population age $13-19$. $^{1}$

Among its varied forms, giant uterine leiomyoma have been met measuring up to $30 \mathrm{~cm}$ in the largest diameter. These myomas were usually solitary leiomyoma picked up in the age 14-18, making the resection easier indispensable even for robotic surgery.4 Multiple myoma is solely reported in one case. ${ }^{3}$

It appears that careful evaluation is necessary to distinguish the tumor from an adnexal lesion, when myoma is suspected in young girl presenting with anaemia, menorrhagia, progressive abdominal enlargement and dysmenorrheal. Despite of the last two findings met in our case, consideration to the diagnosis ran out of our mind. At operation Theatre, we were confronted with the gross findings not imagined before.

Among modalities of treatment in these adolescents with giant myoma of this size, myomectomy, laparoscopic or open laparotomy should be considered, the only valid approach with regards to preferred fertility preserving conservative surgery. One case treated by myomectomy has been blessed by baby. ${ }^{5}$

Leiomyomas in teenagers often exhibit histological features both grossly and microscopically, with extensive hydropic and myxomatous degeneration and areas of prominent vessels, suggesting an angiomatous neoplasm favoring the diagnosis of malignancy, and should be evaluated with extreme caution. The tumour has also been noted to recur. ${ }^{4}$

\section{Conclusion}

Myoma should be considered even in adolescent who present with pelvic mass and congestive dysmenorrhoea, in order to avoid the confrontations of huge multiple myoma unexpectedly so that full preaparedness for surgery can be planned in advance. 
Baral J. , Gurung G. , Mishra S. et. ał3

\section{Reference}

1. Fields KR, Neinstein LS.Uterine myomas in adolescents: case reports and a review of the literature. J Pediatr Adolesc Gynecol. 1996 Nov;9(4):195-8.

2. Grapsa D, Smymiotis V, Hasiakos D, KontogianniKatsarou K, Kondi-Pafiti .Giant uterine leiomyoma simulating an ovarian mass in a 16-year-old girl: a case report and review of the literature. Eur J Gynaecol Oncol. 2006; 27(3):294-6.

3. Tsili AC, Lentoudi ED, Argyropoulou MI, Dalkalitsis N, Batistatou A, Paraskevaidis E, Tsampoulas K. Fibromatous uterus in a 16-year-old girl: a case report. Case Report Med. 2010; 2010:932762.

4. Wright KN, Laufer MR. Leiomyomas in adolescents. Fertil Steril. 2011 Jun;95(7):2434.e15-7.

5. Perkins JD, Hines RS, Prior DS. Uterine leiomyoma in an adolescent female. J Natl Med Assoc. 2009 Jun; 101(6):611-3. 